\title{
Role of the PI3K-AKT-mTOR pathway in hepatitis $B$ virus infection and replication
}

\author{
KUNLUN XIANG and BIN WANG

\begin{abstract}
Key Laboratory of Medical Molecular Virology of The Ministry of Health and The Ministry of
\end{abstract} \\ Education, Shanghai Medical College, Fudan University, Shanghai 200032, P.R. China
}

Received August 12,2017; Accepted November 17, 2017

DOI: $10.3892 / \mathrm{mmr} .2018 .8395$

\begin{abstract}
The replication of hepatitis B virus (HBV) may be modulated by a variety of cell signaling pathways, including the phosphatidylinositol 3-kinase (PI3K)-RAC- $\alpha$ serine/threonine-protein kinase (AKT)-serine/threonine-protein kinase mTOR (mTOR) pathway. The aim of the present study was to determine the regulatory effects of this pathway on the infection and replication of HBV. The results indicated that the HBV entry process may activate the AKT pathway, as demonstrated by AKT phosphorylation in HBV natural infection. However, inhibition of AKT phosphorylation by short-term treatment with AKT inhibitors was unable to block HBV entry, which suggested that AKT activation induced by HBV infection is not essential for viral entry process. Prolonged treatment with PI3K-AKT-mTOR pathway inhibitors markedly promoted HBV replication in HBV replicating and natural infection models. The PI3K-AKT-mTOR pathway was therefore identified to be a negative regulator of HBV replication. These inhibitors enhanced the replication and transcription of HBV in an HBx-dependent way. The results additionally indicated that a PI3K inhibitor, Ly294002, inhibited the secretion of the small surface antigen of HBV in a PI3K-AKT-independent manner. The inhibitor Ly294002 may be used as a tool for the drug development of surface antigen secretion inhibitors.
\end{abstract}

\section{Introduction}

Hepatitis B virus (HBV) is a small DNA virus with a genome size of $3.2 \mathrm{~kb}$. HBV has only four overlapping open reading frames. The $3.5 \mathrm{~kb}$ pre-genomic mRNAs (pgRNAs) and

Correspondence to: Dr Bin Wang, Key Laboratory of Medical Molecular Virology of The Ministry of Health and The Ministry of Education, Shanghai Medical College, Fudan University, 138 Yixueyuan Road, Shanghai 200032, P.R. China

E-mail: bwang3@fudan.edu.cn

Key words: hepatitis B virus, entry, transcription, phosphatidylinositol 3-kinase-RAC- $\alpha$ serine/threonine-protein kinase-serine/threonine-protein kinase mTOR, hepatitis B virus surface antigen pre-core mRNAs (pcRNAs) are produced from juxtaposed promoter sequences (1). The pgRNA serves as the template for viral DNA synthesis and the mRNA for HBV core and polymerase translation. The pcRNA is utilized for the translation of the HBe antigen (Ag) and polymerase. The $2.4 \mathrm{~kb}$ pre-S1/S mRNAs encode the large surface envelope protein of $\mathrm{HBV}$ (LHBs). The pre-S1 region of the large HBsAg is responsible for viral binding and entry processes. The $2.1 \mathrm{~kb}$ pre-S2/S mRNAs encode middle and small envelope proteins (MHBs/SHBs). The concentration of the pre-S1/S mRNA is lower compared with pre-S2/S mRNA in infected hepatocytes (2). The $0.7 \mathrm{~kb} \mathrm{X}$ mRNAs encode the HBx protein, which is the most cryptic protein of HBV with diverse functions $(3,4)$. HBsAg loss and anti-HBs seroconversion are regarded to be a functional cure for chronic HBV infection. HBsAg interacts with plasmacytoid dendritic cells ( $\mathrm{pDCs}$ ) and contributes to pDC impairment (5). High HBV surface antigen concentrations additionally promote $\mathrm{HBV}$-specific T-cell dysfunction by affecting the phenotype and function of peripheral and intrahepatic T cells (6). HBsAg clearance occurs in only a minority of cases, not exceeding $3 \%$ following treatment with nucleoside and nucleotide analogues, and not more than $7.8 \%$ following treatment with pegylated interferon- $\alpha(7,8)$. There is an urgent medical requirement to reduce the serum $\mathrm{HBsAg}$ level and thus reverse T-cell dysfunction.

A number of viruseshave been widely reported to be regulated by class I phosphatidylinositol 3-kinase (PI3K) $(9,10)$. Hepatitis $\mathrm{C}$ virus utilizes the PI3K-RAC- $\alpha$ serine/threonine-protein kinase (AKT) signaling pathway to facilitate viral entry (11). Vaccinia virus and cowpox virus, two members of the poxvirus family, activate the PI3K-AKT pathway to prevent apoptosis, ensuring host survival and viral replication (12). Contradictory to the beneficial role of the PI3K-AKT pathway for the majority of viruses, the PI3K-AKT-serine/threonine-protein kinase mTOR (mTOR) pathway suppresses the replication of HBV and may be partially responsible for the elimination of HBV replication from tumor cells (13). The LHBsAg has been demonstrated to activate the PI3K-AKT pathway and to act as a tumorigenesis factor (14). HBx was reported to activate the PI3K-AKT pathway and act as a balancing factor between HBV replication and cell survival (15-17). LY294002 and wortmannin are first generation compounds with highly potent PI3K-inhibiting properties. The AKT1/2 inhibitor (AKTi) specifically inhibits pleckstrin homology domain-containing kinases. Rapamycin is 
a prototypical inhibitor of mTOR complex 1 (mTORC1) (18). However, prolonged treatment with high concentrations of rapamycin inhibits mTOR2 and AKT activation (19). In the present study, the role of PI3K-AKT-mTOR signaling inhibitors in the infection and replication of HBV was investigated.

\section{Materials and methods}

Cell culture. HepG2 (American Type Culture Collection, Manassas, VA, USA; HB-8065) and Huh7 (JCRB Cell Bank, National Institutes of Biomedical Innovation, Health and Nutrition, Osaka, Japan; 0403) cells were cultured in Dulbecco's modified Eagle's medium/F12 (Life Technologies; Thermo Fisher Scientific, Inc., Waltham, MA, USA) supplemented with $10 \%$ fetal bovine serum (Life Technologies; Thermo Fisher Scientific, Inc.), $2 \mathrm{mM}$ L-glutamine, $100 \mathrm{U} / \mathrm{ml}$ penicillin, and $100 \mu \mathrm{g} / \mathrm{ml}$ streptomycin, at $37^{\circ} \mathrm{C}$ in humidified air containing 5\% $\mathrm{CO}_{2}$. For HepG2.2.15, $300 \mu \mathrm{g} / \mathrm{ml}$ geneticin (Invitrogen; Thermo Fisher Scientific, Inc.) was added. HepAD38 (American Type Culture Collection; CRL-12077) is tetracycline-inducible HBV-producing cell line, in which $\mathrm{HBV}$ expression is suppressed by tetracycline. Tetracylcine (cat no. 250163) and entecavir (cat no. SML1103) were purchased from Sigma-Aldrich (Merck KGaA, Darmstadt, Germany). The cell culture of HepaRG cells (cat no. HPR101; Biopredic International, Saint Grégoire, France) and primary human hepatocytes (Bioreclamation IVT, Hicksville, NY, USA) was performed as previously described $(20,21)$. The cells seeded into 12-well and 96-well plates were at a density of $2 \times 10^{5}$ and $3 \times 10^{4}$, respectively, if not explicitly stated otherwise. The cells were infected with 50 genome equivalents of purified HBV virus from HepAD38 cells, constituted in $4 \%$ polyethylene glycol (PEG; cat no. P1458, Sigma-Aldrich, Merck KGaA) and $1 \%$ dimethyl sulfoxide (DMSO). After $16 \mathrm{~h}$ incubation at $37^{\circ} \mathrm{C}$, cells were washed three times with PBS and replenished with William's E medium (cat no. 32551-020; Life Technologies, Thermo Fisher Scientific, Inc.) supplemented with primary hepatocyte maintenance supplements (cat no. CM4000; Life Technologies; Thermo Fisher Scientific, Inc.) for primary human hepatocyte cell culture, or additives (cat no. ADD710; Biopredic International, Saint Grégoire, France) for HepaRG cells. DMSO, (cat no. D2650) and PI3K-AKT-mTOR inhibitors Ly294002 (cat no. L9908), AKTi (cat no. A6730) and rapamycin (cat no. V900930) were purchased from Sigma-Aldrich (Merck $\mathrm{KGaA}$ ). The inhibitors were dissolved in DMSO and diluted in the William's E or DMEM/F12 medium. The final concentration of DMSO was $1 \%$. Control cells were treated with DMSO alone. In HBV natural infection, Ly294002 was either added $30 \mathrm{~h}$ prior to infection or $30 \mathrm{~h}$ subsequently. In HBV replicating cells, the inhibitors were treated for 4-5 days to monitor the effect of PI3K-AKT-mTOR inhibitors on HBV replication.

Plasmid construction and transient transfection. Plasmid pBR322-HBV1.3ayw, which was kindly provided by Dr. Qiang Deng in Pasteur Institute of Shanghai, contained 1.3-mer length of HBV genome. Small HBV surface antigen (SHBs; spanning nucleotide 157 to nucleotide 837 of HBV ayw genome) were cloned into pcDNA3.1 (+) vector (cat no. V79020; Invitrogen, Thermo Fisher Scientific, Inc.) with KpnI and XhoI. HBx (spanning nucleotide 1376 to nucleotide 1840 of HBV ayw genome) were cloned into pLVX-IRES-tdTomato vector (cat no. 631238; Clontech Laboratories, Inc., Mountainview, CA, USA) with XhoI and BamHI. The restriction enzymes were purchased from New England BioLabs, Inc. (Ipswich, MA, USA). To transduce HBx, the HBx-pLVX-IRES-tdTomato plasmid was used to produce lentivirus by NovoBio (Novobio Scientific, Inc., Shanghai, China). One day before transfection, $1 \times 10^{5}$ cells were seeded into each well of 12-well plates. Plasmids ( $1 \mu \mathrm{g})$ and $3 \mu \mathrm{l} \mathrm{X-tremeGENE}{ }^{\mathrm{TM}}$ HP DNA transfection reagent (cat no. 06366236001; Roche Diagnostics GmbH, Mannheim, Germany) were diluted in $200 \mu$ l Opti-MEM medium (cat no. 31985062; Life Technologies, Thermo Fisher Scientific, Inc.). Incubated for $15 \mathrm{~min}$ at room temperature and added the transfection complex to the cells in a dropwise manner. After $6 \mathrm{~h}$ incubation at $37^{\circ} \mathrm{C}$, cells were replenished with $1 \mathrm{ml}$ cell culture medium. The cells were incubated for 5 days post transfection with or without the presence of PI3K-AKT-mTOR inhibitors.

DNA/RNA extraction and quantification. The DNA and RNA were extracted using a DNA Blood Mini kit (Qiagen GmbH, Hilden, Germany) and TRIzol reagent (Thermo Fisher Scientific, Inc.), respectively. The RNAs were reverse transcribed using a cDNA synthesis kit (Bio-Rad Laboratories, Inc., Hercules, CA, USA). Plasmid pBR322-HBV1.3ayw, which contained 1.3mer HBV genome, was used as HBV DNA standard for PCR. Quantitative polymerase chain reaction (qPCR) analysis was performed using TaqMan qPCR Master Mix reagent (Roche Diagnostics, Basel, Switzerland). The primers used for HBV DNA quantification were: HBX forward, 5'-cccgtctgtgcettctcatc-3'; HBX reverse, 5'-tcggtcgtt gacattgctga-3'; HBX probe, 5'-FAM+tcgcttcacctctgcacgtcgc + Black Hole Quencher ${ }^{\circledR}-3^{\prime}$. The PCR cycling program was performed as follows: Denaturation at a temperature of $95^{\circ} \mathrm{C}$ for $10 \mathrm{~min}$, followed by 45 cycles of amplification at a denaturation temperature of $95^{\circ} \mathrm{C}$ for $10 \mathrm{sec}$, an annealing temperature of $60^{\circ} \mathrm{C}$ for $10 \mathrm{sec}$, and an extension temperature of $72^{\circ} \mathrm{C}$ for $15 \mathrm{sec}$. The cycle of quantification $(\mathrm{Cq})$ was obtained to determine the absolute quantification of HBV DNAs using a standard curve. The standard curve was generated by plotting Cq values vs. the concentrations of HBV DNA standard with the analysis method 'Abs Quant/2nd Derivative Max for All Samples' of the LightCycler480 software (Roche Diagnostics GmbH, Mannheim, Germany). HBV DNA copy numbers were calculated from $\mathrm{Cq}$ values fit on the HBV plasmid DNA standard curve by the LightCycler480 software.

Agarose gel electrophoresis of viral particles. Cells seeded in 12-well plates were lysed in $0.5 \mathrm{ml}$ lysis buffer $(50 \mathrm{mM}$ Tris- $\mathrm{HCl}$ [pH 7.5], $150 \mathrm{mM} \mathrm{NaCl}, 0.5 \%$ CA-630, supplemented with a protease $\&$ phosphatase inhibitor cocktail, cat no. 78440, Thermo Fisher Scientific, Inc.) and treated with same procedure as previously described (22). The HBV capsids were blotted to enhanced chemiluminescence membranes (GE Healthcare, Chicago, IL, USA) by capillary transfer. The membranes were blocked with $10 \%$ non-fat milk in TBS- $0.1 \%$ Tween 20 for $30 \mathrm{~min}$. HBV capsids were detected with primary anti-core antibody (cat no. B0586; Dako, Agilent Technologies, Inc., Santa Clara, CA, USA). The membrane was incubated with $1: 1,000$ diluted primary antibody at $4^{\circ} \mathrm{C}$ with gentle 
A

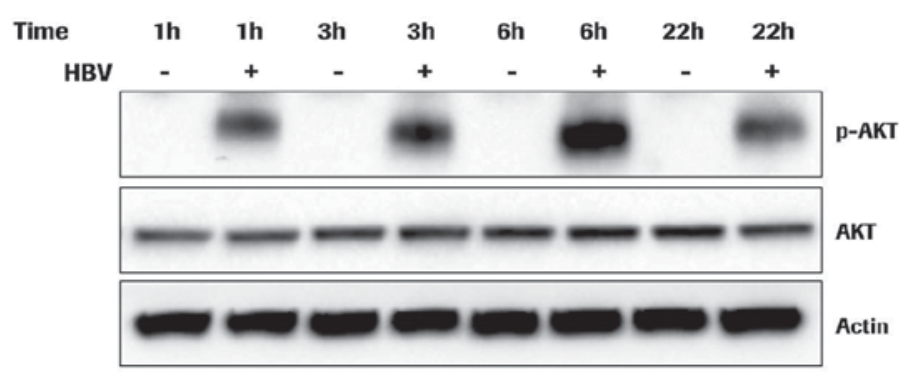

C

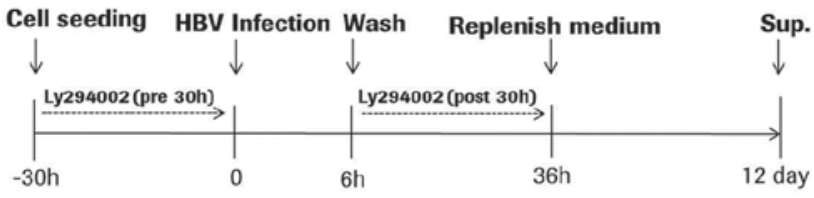

B

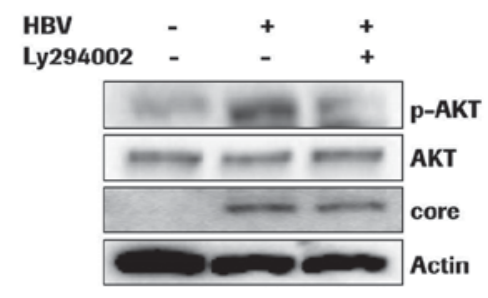

D

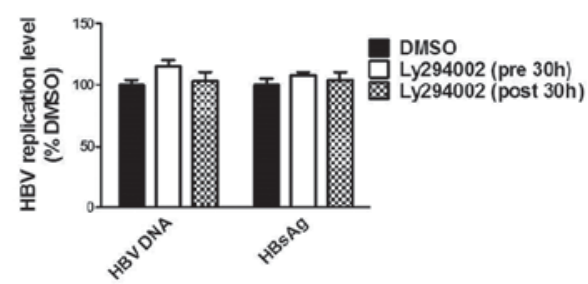

Figure 1. HBV entry process activates the AKT pathway. (A) Differentiated HepaRG cells were infected with HBV at 50 genome equivalents/cell. The AKT, p-AKT and $\beta$-actin levels at different time points were detected by western blotting. (B) In PHHs, Ly294002 inhibited AKT phosphorylation induced by HBV infection at $36 \mathrm{~h}$ post-HBV inoculation. The HBV core protein level was not affected by AKT inhibition with Ly294002. (C) The PHHs were seeded $30 \mathrm{~h}$ prior to HBV infection. Ly 294002 was added either at $30 \mathrm{~h}$ prior to or following HBV infection. The cell culture medium was replenished every 3 days. The supernatant was collected on day 12 for viral DNA and HBsAg quantification. (D) HBV DNAs and HBsAg in the cell culture medium were evaluated at 12 days post-infection. The Ly294002 was either added $30 \mathrm{~h}$ prior to infection or $30 \mathrm{~h}$ subsequently. The Ly294002 was withdrawn from the cell culture medium and the cells were cultured without Ly294002 until day 12 post-infection. PHH, primary human hepatocyte; AKT, RAC- $\alpha$ serine/threonine-protein kinase; HBV, hepatitis B virus; DMSO, dimethyl sulfoxide; p, phosphorylated; Ag, antigen.

shaking overnight. Following washing three times with TBST, the membrane was incubated with 1:2,000 diluted anti-rabbit antibody (cat no. 656120; Thermo Fisher Scientific, Inc.) at room temperature for $1 \mathrm{~h}$. The blot was incubated with horse radish peroxidase (HRP) substrate (cat no. 34075; Thermo Fisher Scientific, Inc.) and imaged using the ChemiDoc touch imaging system (Bio-Rad Laboratories, Inc.). The HBV capsid DNA was transferred to nylon membranes and hybridized with digoxigenin-labeled DNA probes according to the standard procedure of southern blotting.

Southern and northern blotting. The HBV capsid DNAs were hybridized with DNA probes and detected by alkaline phosphatase-conjugated anti-digoxigenin antibody (23). For RNA detection, $5 \mu \mathrm{g}$ tRNAs were resolved on a $1.5 \%$ agarose gel containing $0.66 \mathrm{M}$ formaldehyde, transferred onto nylon membranes and detected with HBV RNA probes (22).

Western blotting. Cells were washed once with Tris-buffered saline (TBS) and lysed with radioimmunoprecipitation assay lysis buffer (cat no. 89,900; Thermo Fisher Scientific, Inc.) supplemented with a phosphatase and protease inhibitor cocktail (cat no. 78440; Thermo Fisher Scientific, Inc.). Total protein concentrations in the lysate were measured using a bicinchoninic acid protein assay kit (cat no. 23225; Thermo Fisher Scientific, Inc.). Cell lysates were mixed with 4x SDS loading buffer (cat no. NP0007; Thermo Fisher Scientific, Inc.) and $10 \mathrm{mM}$ DTT, and were boiled for $5 \mathrm{~min}$ at $95^{\circ} \mathrm{C}$. A total of $5 \mu \mathrm{g}$ of protein was loaded onto each lane. The samples were subjected to 4-12\% SDS-PAGE (cat no. NP0322; Thermo Fisher Scientific, Inc.) and were then transferred to nitrocellulose membranes (cat no. IB23001; Thermo Fisher Scientific,
Inc.). The membranes were blocked with $10 \%$ nonfat milk (cat no. 9999; Cell Signaling Technology, Inc., Danvers, MA, USA) in TBS- $0.1 \%$ Tween 20 for at least $30 \mathrm{~min}$ and were then incubated with the indicated primary antibodies. The primary antibodies used were as follows: $\beta$-actin (cat. no. A3854; Sigma-Aldrich; Merck KGaA), HBV core (cat. no. B0586; Dako; Agilent Technologies, Inc., Santa Clara, CA, USA), AKT (cat. no. 9272; Cell Signaling Technology, Inc., Danvers, MA, USA), p-AKT (cat. no. 9275; Cell Signaling Technology, Inc.), HBsAg (cat. no. 30R-AH018; Fitzgerald Industries International, North Acton, MA, USA), and HBx (kindly provided by Roche Innovation Centre Shanghai, Shanghai, China). The membrane was incubated with 1:1,000 diluted primary antibody at $4^{\circ} \mathrm{C}$ with gentle shaking overnight. The secondary antibodies, including anti-rabbit antibody (cat no. 656120) and anti-mouse antibody (cat no. 32430) were purchased from Thermo Fisher Scientific, Inc. Following washing three times with TBS, the membrane was incubated with 1:2,000 diluted secondary antibody at room temperature for $1 \mathrm{~h}$. The blot was incubated with HRP substrate (cat no. 34075; Thermo Fisher Scientific, Inc.) and imaged using the ChemiDoc touch imaging system (Bio-Rad Laboratories, Inc.).

Immunofluorescence. HepAD38 cells $\left(8 \times 10^{4}\right)$ were seeded into each well of chamber slides (cat. no. 177445; Thermo Fisher Scientific, Inc.). Following treatment with the inhibitors for three days, cells were fixed with $4 \%$ paraformaldehyde at room temperature for $20 \mathrm{~min}$. The samples were incubated for 10 min with PBS containing either $0.5 \%$ Triton X-100 at room temperature for $10 \mathrm{~min}$, and then were washed three times with PBS for $5 \mathrm{~min}$. Then, samples were incubated with HBsAg antibody (1:100; cat. no. 18-0023; Invitrogen; Thermo Fisher 

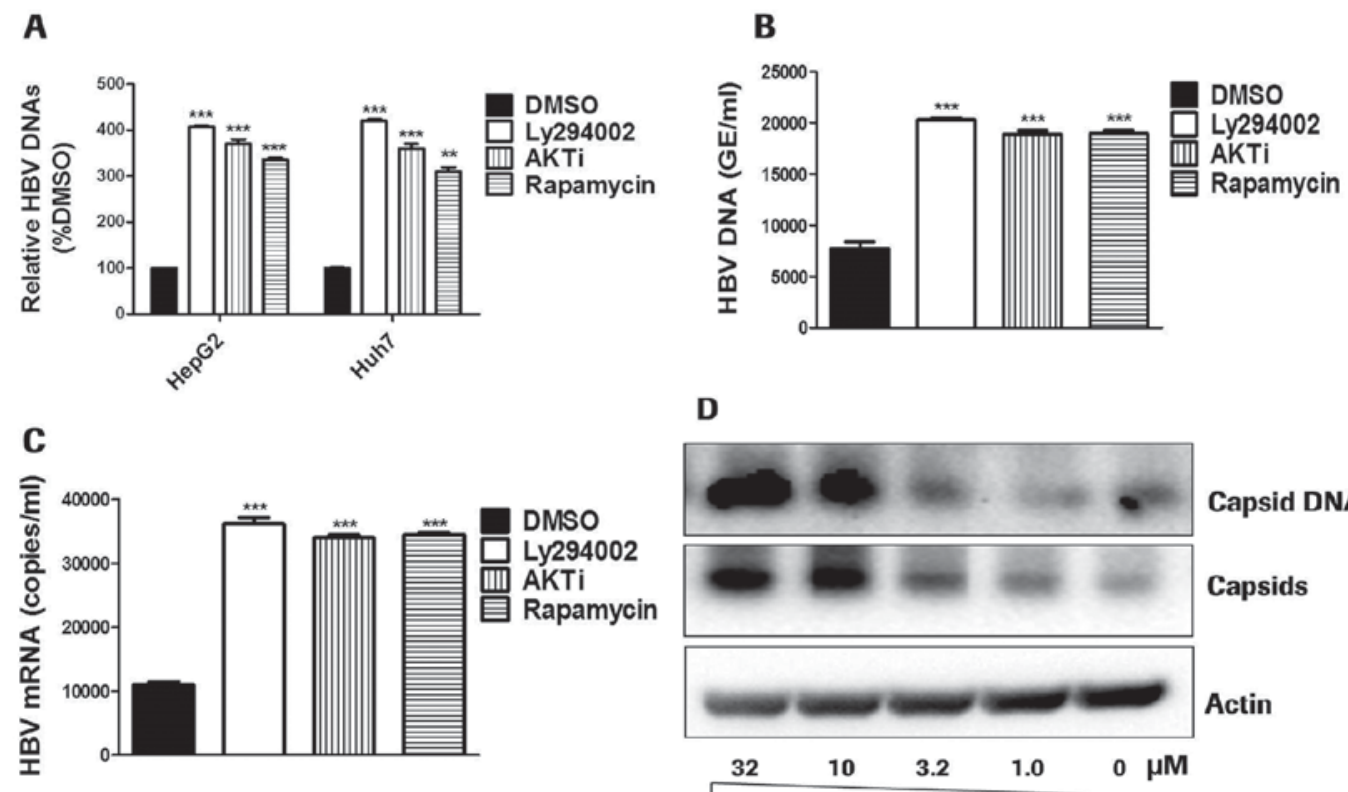

D
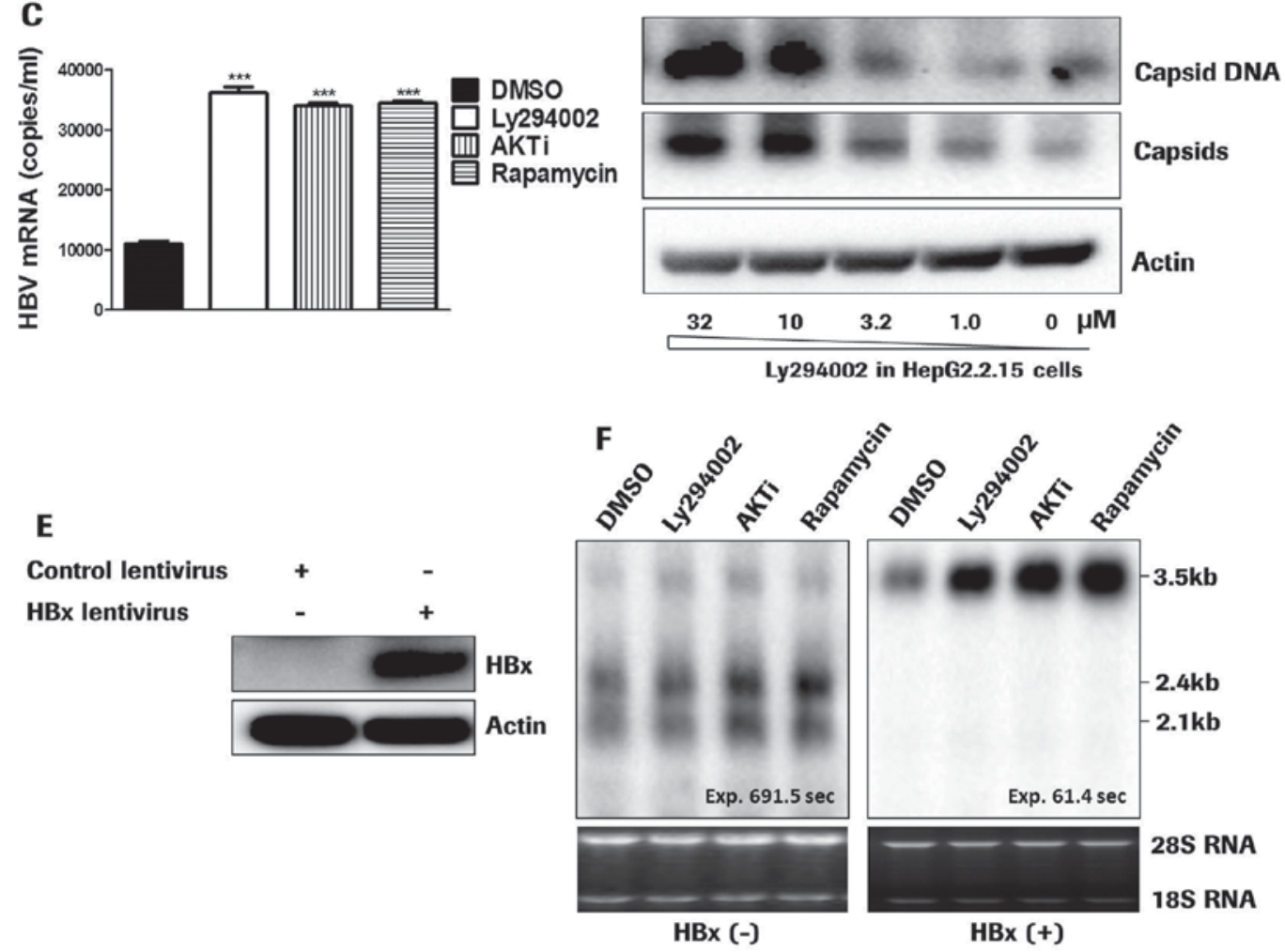

Figure 2. PI3K-AKT inhibitors promote HBV replication. (A) pHBV1.3 plasmid-transfected HepG2 and Huh7 cells were treated with PI3K-AKT inhibitors for 5 days. HBV DNA in the supernatants was detected by quantitative polymerase chain reaction analysis. (B) In HBV-infected dHepaRG cells, the prolonged presence of PI3K-AKT inhibitors between days 5 and 12 increased the HBV DNA level. (C) HBV RNAs were promoted in dHepaRG cells treated with PI3K-AKT inhibitors. (D) In HepG2.2.15 cells, the intracellular capsids were detected with agarose gel electrophoresis of viral particles. Capsid DNA was detected by Southern blotting. Treatment with Ly294002 for 5 days increased HBV capsids and capsid DNAs in a dose-dependent manner. (E) In enticavirand tetracycline-treated HepAD38 cells, HBx expression was detected by western blotting. (F) HBV RNA transcription in HepAD38 cells was evaluated via northern blotting. The HBx gene was introduced into HepAD38 cells via lentiviral transduction. The cells were treated with PI3K-AKT-mTOR inhibitors for 5 days. $18 \mathrm{~S}$ and $28 \mathrm{~S}$ ribosomal RNAs were used as the loading control. ${ }^{* *} \mathrm{P}<0.01,{ }^{* * *} \mathrm{P}<0.001$ vs. DMSO. PI3K, phosphatidylinositol 3-kinase; AKT, RAC- 0 serine/threonine-protein kinase; HBV, hepatitis B virus; DMSO, dimethyl sulfoxide; AKTi, AKT1/2 inhibitor; GE, genome equivalents.

Scientific, Inc.) in PBST buffer supplemented with 5\% normal serum for $2 \mathrm{~h}$ at room temperature, and washed three times with PBST buffer for $5 \mathrm{~min}$. Samples were then incubated with goat anti-mouse antibody (1:400; cat. no. A28175; Invitrogen; Thermo Fisher Scientific, Inc.) at room temperature for $30 \mathrm{~min}$ in the dark and washed thrice with PBST buffer for $5 \mathrm{~min}$. Samples were incubated with $100 \mathrm{nM}$ DAPI in PBS buffer at room temperature for $5 \mathrm{~min}$, and then cells were washed three times with PBS for $5 \mathrm{~min}$ in the dark. The cells were visualized with a Zeiss confocal microscope (Zeiss GmbH, Jena, Germany) at 400x magnification.

Chemiluminescence immunoassay (CLIA). HBsAg and $\mathrm{HBeAg}$ in the culture medium and cell lysate were tested using
Autobio CLIA kits (Autobio Co., Ltd., Zhengzhou, China; cat. nos. CL0310-2 and CL0310-2 for HBsAg and HBeAg, respectively), according to the manufacturer's protocol.

Statistical analysis. HBV DNA/RNA and HBsAg levels were quantified by PCR and CLIA assay respectively. Data represent the mean \pm standard deviation of experiments performed in triplicate, and are presented as absolute copy numbers or relative to control (cells treated with DMSO). One-way analysis of variance was used with Dunn's multiple comparisons test (GraphPad Prism version 7.03 software; GraphPad Software, Inc., La Jolla, CA, USA) to compare the different groups in the present study. $\mathrm{P}<0.05$ was considered to indicate a statistically significant difference. 
A

Ly294002 in HepG2.2.15 cells

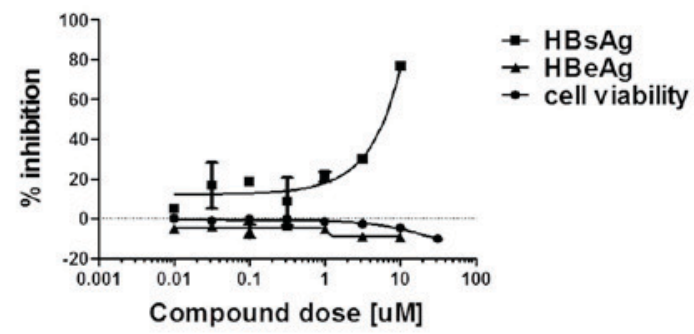

Rapamycin in HepG2.2.15 cells

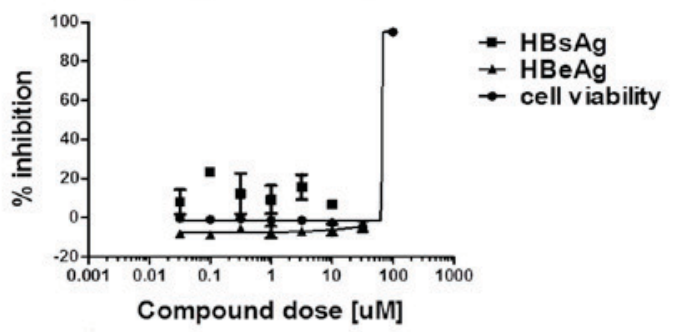

B
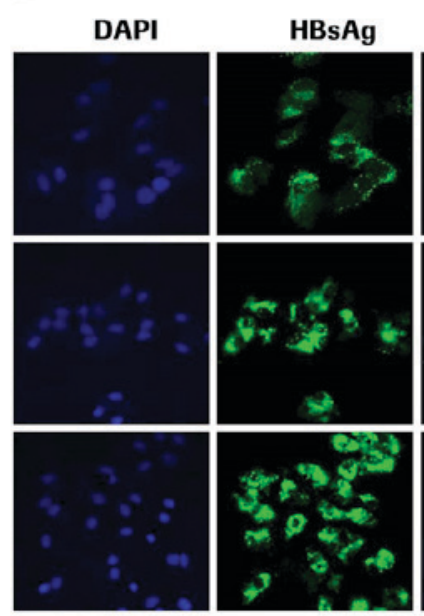

D

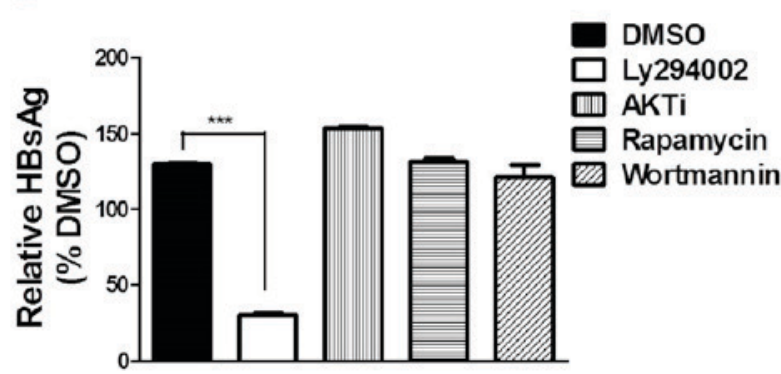

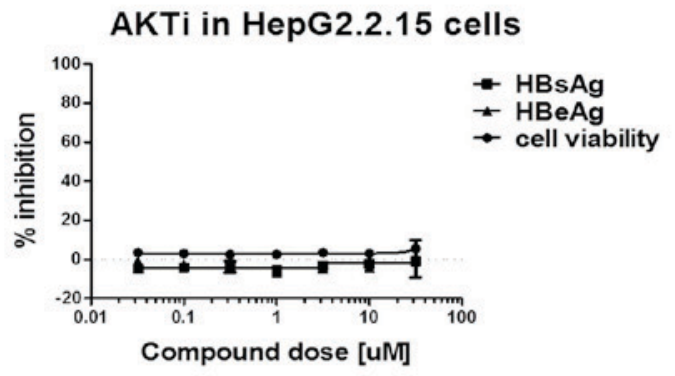

Wortmannin in HepG2.2.15 cells

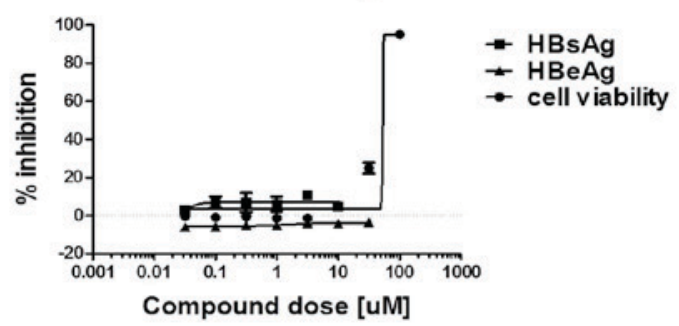

C

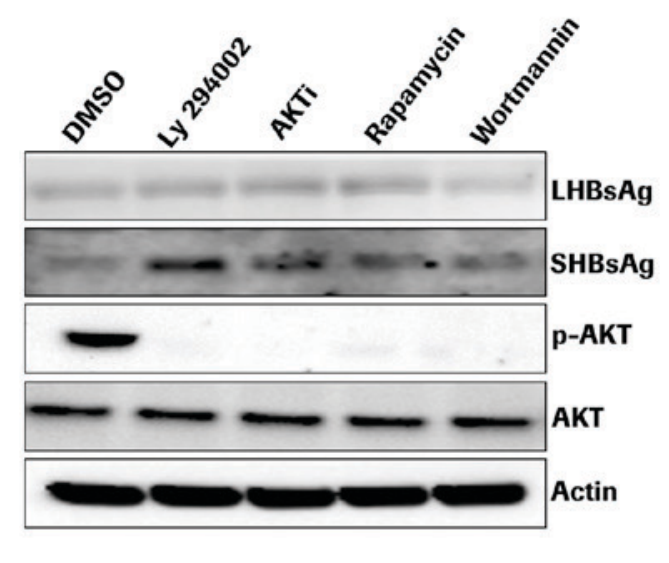

$\mathbf{E}$

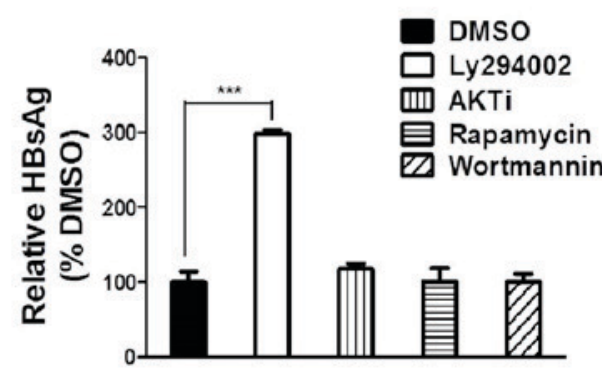

Figure 3. Ly294002 inhibits small HBsAg secretion in a PI3K-AKT-mTOR pathway-independent manner. (A) For HepG2.2.15 cells, HBsAg and HBeAg were detected via chemiluminescence immunoassays. Cell viability was detected by Cell Counting Kit-8 assay. (B) HepAD38 cells were treated with Ly294002 for 4 days. The cells were immunostained with the antibody to HBsAg (green color) and counterstained with DAPI (blue color). The images were acquired on a confocal microscope at 400x magnification. (C) HepG2.2.15 cells were treated with inhibitors for 4 days. HBsAg, AKT and $\beta$-actin were detected via western blotting. (D) In SHBsAg plasmid-transfected HepG2 cells, treatment with Ly294002 decreased extracellular SHBsAg levels. (E) In SHBsAg plasmid-transfected HepG2 cells, Ly294002 promoted intracellular SHBsAg levels. ${ }^{* * *} \mathrm{P}<0.001$. Ag, antigen; PI3K, phosphatidylinositol 3-kinase; AKT, RAC- $\alpha$ serine/threonine-protein kinase; mTOR, serine/threonine-protein kinase mTOR; HB, hepatitis B; DMSO, dimethyl sulfoxide; p, phosphorylated; LHB, large surface envelope protein; SHB, small surface envelope protein; AKTi, AKT1/2 inhibitor.

\section{Results}

$H B V$ entry process activates the AKT pathway. In order to evaluate the AKT phosphorylation induced by the HBV entry process, differentiated HepaRG cells were utilized for HBV infection, and cell lysates were collected at different time 
points post-HBV infection. HBV cellular entry transiently activated AKT and resulted in AKT phosphorylation at threonine 308 as early as $1 \mathrm{~h}$ post-HBV infection (Fig. 1A). AKT phosphorylation peaked at $6 \mathrm{~h}$ post-HBV infection and gradually decreased at $22 \mathrm{~h}$ post-infection.

Furthermore, AKT activation was transiently inhibited by the PI3K inhibitor Ly294002 at $10 \mu \mathrm{M}$, at which concentration the AKT phosphorylation was markedly inhibited. HBV entry activated AKT at $36 \mathrm{~h}$ post-viral infection in primary human hepatocytes, as hypothesized (Fig. 1B). Inhibition of AKT phosphorylation exerted no effect on HBV cellular entry, as the HBV core protein level in the cell lysate was not affected at $36 \mathrm{~h}$ post-HBV infection (Fig. 1B). The results demonstrated that although the HBV cellular entry process was able transiently activate the AKT pathway, AKT phosphorylation was not essential for HBV entry compared with a variety of viruses (11-13). The HBV infection and compound treatment procedure is illustrated in Fig. 1C. The PI3K inhibitor Ly294002 was applied for $30 \mathrm{~h}$ prior to or following HBV infection. Quantification of HBV DNA and HBsAg levels at 12 days post-infection additionally indicated that transient inhibition of AKT phosphorylation with $10 \mu \mathrm{M}$ Ly294002 had no effect on the HBV entry process (Fig. 1D).

Prolonged treatment with PI3K-AKT-mTOR pathway inhibitors promotes $H B V$ replication. First of all, the effect of PI3K-AKT-mTOR inhibitors was evaluated in HBV plasmid transfected HepG2 and Huh7 cells. All three inhibitors, including Ly294002, AKTi and rapamycin, promoted HBV DNA levels in the supernatant following 5 days of treatment (Fig. 2A). In HBV infected dHepaRG cells, prolonged treatment with PI3K-AKT inhibitors increased the extracellular HBV DNAs in addition to the intracellular HBV RNAs (Fig. 2B and C). The prolonged effect of Ly294002 on HBV replication was further confirmed in HepG2.2.15 cells. The PI3K inhibitor Ly294002 dose-dependently increased the levels of HBV capsids and capsid DNAs in the cell lysates (Fig. 2D). These results were consistent with previous studies $(13,15)$, and demonstrated that the PI3K-AKT-mTOR pathway negatively regulates $\mathrm{HBV}$ replication.

In addition, HepAD38 cells were treated without tetracycline for 8 days to accumulate intracellular covalently closed circular (ccc)DNAs. The cells were treated with $200 \mathrm{nM}$ entecavir and tetracycline for 5 days to inhibit reverse transcription and transcription from integrated HBV DNA, respectively. Transcripts from episomal cccDNA were not affected by treatment with entecavir and tetracycline. Lentiviruses were utilized to introduce HBx into HepAD38 cells. The effect of the PI3K-AKT-mTOR signaling pathway inhibitors on HBV transcription was evaluated. In entecavir- and tetracycline-treated HepAD38 cells, HBx expression was undetectable by western blotting (Fig. 2E). The PI3K-AKT-mTOR pathway inhibitors promoted HBV $3.5 \mathrm{~kb}$ pgRNA transcription in HBx-expressing cells, and exerted no effect on pgRNA transcription in HBx negative cells. The enhanced transcription was specific to $3.5 \mathrm{~kb}$ mRNAs, as it exerted no impact on the transcription of $2.4 \mathrm{~kb}$ and $2.1 \mathrm{~kb} \mathrm{~S}$ mRNAs (Fig. 2F). The results demonstrated that the PI3K-AKT-mTOR pathway inhibitors promoted HBV $3.5 \mathrm{~kb}$ pgRNA transcription in an HBx-dependent manner.
Ly294002 inhibits small HBsAg secretion in a PI3K-AKT pathway-independent manner. HepG2.2.15 cells were treated with PI3K-AKT-mTOR inhibitors for 4 days. Ly294002 was observed to be the only inhibitor which was able to decrease HBsAg levels in the cell culture medium (Fig. 3A). It was subsequently identified that Ly294002 was the only compound increasing intracellular HBsAg in HepG2.2.15 cells (data not shown). The results indicated that Ly294002 may block HBsAg secretion processes.

This HBsAg secretion inhibition capability of the PI3K inhibitor Ly294002 was further confirmed by immunofluorescence staining of HBsAg in HepAD38 cells (Fig. 3B). The results also indicated that Ly294002 blocked HBsAg secretion and increased the intracellular HBsAg level in a dose dependent manner. Western blot detection of intracellular HBsAg demonstrated that all of the four inhibitors intensively inhibited AKT phosphorylation. However, only Ly294002 blocked small HBsAg (SHBs) secretion, and exerted no effect on large HBsAg (Fig. 3C). In SHB plasmid-transfected HepG2 cells, following treatment with Ly294002, AKTi, rapamycin and wortmannin, only Ly294002 inhibited the secretion of sHBsAg (Fig. 3D and E). These results further demonstrated that the PI3K inhibitor Ly294002 may specifically inhibit the secretion of small HBsAg in a PI3K-AKT-mTOR pathway-independent manner.

\section{Discussion}

In the present study, the regulatory effect of the PI3K-AKT-mTOR pathway in HBV infection and replication was investigated using different in vitro cell culture systems. The role of this pathway in hepatocellular carcinoma (HCC) was not evaluated. In addition, certain cell lines used in the present study, including HepG2, HepG2.2.15 and HepAD38, are actually hepatoblastoma cells and are not unsuitable for HCC study. The role of AKT activation in the HBV entry process was evaluated in a HBV natural infection model. The results indicated that AKT phosphorylation at threonine 308 was markedly induced by HBV entry as early as $1 \mathrm{~h}$ post-HBV inoculation. Blocking AKT activation during the first $30 \mathrm{~h}$ of the HBV entry process via treatment with Ly294002 did not affect HBV entry, as short-term treatment with AKT inhibitors exerted no effect on HBV core levels at $30 \mathrm{~h}$ post-infection, nor on HBV DNA production at day 12. These results indicated that the HBV entry process activated the AKT pathway, demonstrated by AKT phosphorylation in HBV natural infection. However, inhibition of AKT phosphorylation via short-term treatment with AKT inhibitors was unable to inhibit HBV entry, suggesting that AKT activation induced by $\mathrm{HBV}$ infection is not essential for the viral entry process.

The PI3K-AKT-mTOR signaling pathway has been observed to negatively regulate HBV replication in the HepG2 cell line $(13,15)$. The present study used HBV natural infection to study the role of this multifunctional pathway in the HBV entry, transcription and HBsAg secretion processes. The results demonstrated that the PI3K-AKT inhibitors strongly increased HBV DNA levels by enhancing HBV $3.5 \mathrm{~kb}$ pgRNA transcription in an $\mathrm{HBx}$-dependent manner. As the HBeAg in the cell culture medium remained unchanged in HepAD38 cells (data not shown), the dramatically increased $3.5 \mathrm{~kb}$ 
RNAs were almost pgRNAs, not the $3.5 \mathrm{~kb}$ pcRNAs which encode HBeAg. This result was consistent with previous studies $(13,15)$, which indicated that the PI3K-AKT-mTOR pathway was able to negatively regulate HBV replication and balance cell survival. However, these inhibitors only promoted pgRNA, and not S mRNA transcription, which suggested that the transcriptional enhancement was specific to pgRNAs.

To the best of our knowledge, the present study was the first to utilize a HBV natural infection model to study the role of the PI3K-AKT-mTOR pathway in HBV infection and replication. In HBV-infected primary human hepatocytes (Fig. 1D), Ly294002 was added at $30 \mathrm{~h}$ prior to or following HBV inoculation to inhibit the AKT phosphorylation induced by HBV. The short-term existence of the PI3K inhibitor Ly294002 exerted no influence on the HBV entry process. This result indicated that, although HBV infection may trigger AKT activation, the AKT activation induced by HBV infection is not essential for HBV entry. In the HBV replication and infection models (Fig. 2A-C), to evaluate the influence of PI3K-AKT-mTOR inhibitors on the long-term replication of $\mathrm{HBV}$, including HBV transcription, reverse transcription and expression, these inhibitors were applied for at least 5 days. The results suggested that the inhibitors promoted HBV replication in different HBV replicating systems. Further investigation may be required to identify whether off-target effects of PI3K-AKT-mTOR inhibitors contribute to the regulation of HBV replication.

In addition, the PI3K inhibitor Ly294002 blocked small HBsAg secretion in a PI3K-AKT independent manner, and thus decreased the HBsAg level in the cell culture medium. The other PI3K-AKT pathway inhibitors, AKTi, rapamycin and wortmannin, had no effect on HBsAg secretion. Decreased HBsAg levels in patient serum may be of benefit in reversing HBV-specific T-cell dysfunction and pDC impairment. As mentioned previously, low HBsAg levels in patient serum confer an increased HBsAg clearance potential (7). Ly294002 may be used as a tool for the development of drugs which are able to decrease the HBsAg level in patient serum. Combination treatment with $\mathrm{HBsAg}$ inhibitors and immune-modulators may improve anti-HBV therapy (24).

\section{Acknowledgements}

$\mathrm{K}$. Xiang is supported by the education assistance program of Roche Innovation Center Shanghai (RICS). The authors would like to thank RICS for providing reagents and materials for the present study.

\section{References}

1. $\mathrm{Yu} X$ and Mertz JE: Promoters for synthesis of the pre-C and pregenomic mRNAs of human hepatitis B virus are genetically distinct and differentially regulated. J Virol 70: 8719-8726, 1996.

2. Raney AK and McLachlan A: Characterization of the hepatitis B virus large surface antigen promoter Sp1 binding site. Virology 208: 399-404, 1995.

3. Gearhart TL and Bouchard MJ: The hepatitis B virus X protein modulates hepatocyte proliferation pathways to stimulate viral replication. J Virol 84: 2675-2686, 2010.

4. Benhenda S, Cougot D, Buendia MA and Neuveut C: Hepatitis B virus $\mathrm{X}$ protein molecular functions and its role in virus life cycle and pathogenesis. Adv Cancer Res 103: 75-109, 2009.
5. Woltman AM, Op den Brouw ML, Biesta PJ, Shi CC and Janssen HL: Hepatitis B virus lacks immune activating capacity, but actively inhibits plasmacytoid dendritic cell function. PLoS One 6: e15324, 2011.

6. Schurich A, Khanna P, Lopes AR, Han KJ, Peppa D, Micco L, Nebbia G, Kennedy PT, Geretti AM, Dusheiko G and Maini MK: Role of the coinhibitory receptor cytotoxic $\mathrm{T}$ lymphocyte antigen-4 on apoptosis-Prone CD8 T cells in persistent hepatitis B virus infection. Hepatology 53: 1494-1503, 2011.

7. Lok AS and McMahon BJ: Chronic hepatitis B: Update 2009. Hepatology 50: 661-662, 2009.

8. European Association For The Study Of The Liver: EASL clinical practice guidelines: Management of chronic hepatitis B virus infection. J Hepatol 57: 167-185, 2012.

9. Cooray S: The pivotal role of phosphatidylinositol 3-kinase-Akt signal transduction in virus survival. J Gen Virol 85: 1065-1076, 2004.

10. Buchkovich NJ, Yu Y, Zampieri CA and Alwine JC: The TORrid affairs of viruses: Effects of mammalian DNA viruses on the PI3K-Akt-mTOR signalling pathway. Nat Rev Microbiol 6: 266-275, 2008.

11. Liu Z, Tian Y, Machida K, Lai MM, Luo G, Foung SK and Ou JH: Transient activation of the PI3K-AKT pathway by hepatitis C virus to enhance viral entry. J Biol Chem 287: 41922-41930, 2012.

12. Soares JA, Leite FG, Andrade LG, Torres AA, De Sousa LP, Barcelos LS, Teixeira MM, Ferreira PC, Kroon EG, Souto-Padrón T and Bonjardim CA: Activation of the PI3K/Akt pathway early during vaccinia and cowpox virus infections is required for both host survival and viral replication. J Virol 83: 6883-6899, 2009.

13. Guo H, Zhou T, Jiang D, Cuconati A, Xiao GH, Block TM and Guo JT: Regulation of hepatitis B virus replication by the phosphatidylinositol 3-kinase-akt signal transduction pathway. J Virol 81: 10072-10080, 2007.

14. Liu H, Xu J, Zhou L, Yun X, Chen L, Wang S, Sun L, Wen Y and $\mathrm{Gu}$ J: Hepatitis B virus large surface antigen promotes liver carcinogenesis by activating the Src/PI3K/Akt pathway. Cancer Res 71: 7547-7557, 2011.

15. Rawat $\mathrm{S}$ and Bouchard MJ: The hepatitis B virus (HBV) $\mathrm{HBx}$ protein activates AKT to simultaneously regulate HBV replication and hepatocyte survival. J Virol 89: 999-1012, 2015.

16. Lee YI, Kang-Park S, Do SI and Lee YI: The hepatitis B virus-X protein activates a phosphatidylinositol 3-kinase-dependent survival signaling cascade. J Biol Chem 276: 16969-16977, 2001.

17. Shih WL, Kuo ML, Chuang SE, Cheng AL and Doong SL: Hepatitis B virus $X$ protein activates a survival signaling by linking SRC to phosphatidylinositol 3-kinase. J Biol Chem 278: 31807-31813, 2003.

18. Zhou Q, Lui VW and Yeo W: Targeting the PI3K/Akt/mTOR pathway in hepatocellular carcinoma. Future Oncol 7: 1149-1167, 2011.

19. Sarbassov DD, Ali SM, Sengupta S, Sheen JH, Hsu PP, Bagley AF, Markhard AL and Sabatini DM: Prolonged rapamycin treatment inhibits mTORC2 assembly and Akt/PKB. Mol Cell 22: 159-168, 2006.

20. Cerec V, Glaise D, Garnier D, Morosan S, Turlin B, Drenou B, Gripon P, Kremsdorf D, Guguen-Guillouzo C and Corlu A: Transdifferentiation of hepatocyte-like cells from the human hepatoma HepaRG cell line through bipotent progenitor. Hepatology 45: 957-967, 2007.

21. Gripon P, Rumin S, Urban S, Le Seyec J, Glaise D, Cannie I, Guyomard C, Lucas J, Trepo C and Guguen-Guillouzo C: Infection of a human hepatoma cell line by hepatitis B virus. Proc Natl Acad Sci USA 99: 15655-15660, 2002.

22. Zhang S, Guo JT, Wu JZ and Yang G: Identification and characterization of multiple TRIM proteins that inhibit hepatitis B virus transcription. PLoS One 8: e70001, 2013.

23. Shi L, Li S, Shen F, Li H, Qian S, Lee DH, Wu JZ and Yang W: Characterization of nucleosome positioning in hepadnaviral covalently closed circular DNA minichromosomes. J Virol 86: 10059-10069, 2012.

24. Lanford RE, Guerra B, Chavez D, Giavedoni L, Hodara VL, Brasky KM, Fosdick A, Frey CR, Zheng J, Wolfgang G, et al: GS-9620, an oral agonist of Toll-like receptor-7, induces prolonged suppression of hepatitis B virus in chronically infected chimpanzees. Gastroenterology 144: 1508-1517, 2013. 